\title{
Renal denervation: potential indications and review of trial data
}

\author{
Author: Claire E Raphael ${ }^{\mathrm{A}}$
}

\section{Renal sympathetic denervation is a novel, endovascular treatment with potential utility in resistant hypertension. First pioneered in humans in 2010 , conflicting data exist regarding the efficacy of this treatment. This article will review the evidence to date, the scientific rationale for sympathetic denervation as a treatment for hypertension and consider other possible conditions where the therapy may be of benefit.}

KEYWORDS: Renal denervation, symplicity, hypertension, renal sympathetic denervation

\section{Introduction}

Renal sympathetic denervation has received much attention since the proof of principle Symplicity HTN-1 trial in 2009, ${ }^{1}$ which demonstrated large reductions in blood pressure following denervation in a cohort of patients with resistant hypertension. Since then, further data have suggested a potential role in other disease processes including heart failure, atrial and ventricular arrhythmia, sleep apnoea and diabetes, with trials ongoing in these areas. However, the Symplicity HTN-3 trial, ${ }^{2}$ published in April 2014, showed no benefit of renal denervation compared with a sham procedure in a large cohort of patients with resistant hypertension. This single trial highlighted the importance of a sham arm, and cast doubt on the future of this procedure in clinical practice.

\section{Renal denervation: a potential endovascular treatment for hypertension?}

Hypertension is a common cause of premature morbidity and mortality worldwide. It is a major risk factor for stroke and coronary artery disease, with each $2 \mathrm{mmHg}$ increase in systolic blood pressure being associated with a $10 \%$ increase in risk of death from stroke and a $7 \%$ increase in death from ischaemic heart disease. ${ }^{3}$ Even with improved monitoring of blood pressure in primary care, approximately $10 \%$ of patients have resistant hypertension, failing to meet treatment targets despite three or more anti-hypertensives, and remain at high risk for future clinical events.

Patients with hypertension commonly have high sympathetic activity, which is largely mediated via the renal efferent and

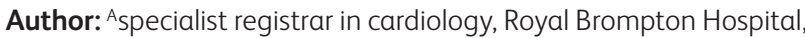
London, UK afferent nerves. Stimulation of the renal efferent nerve causes release of renin from the kidneys and therefore activation of the renin-angiotensin-aldosterone system, with consequent arterial vasoconstriction and salt and water retention. The renal afferent nerves increase activity in response to hypoxia, stretch or renal ischemia. Stimulation of the renal afferent nerves causes feedback via the hypothalamus, and further stimulation of the sympathetic nervous system. The net result of all of these mechanisms is to increase the circulating volume of blood and to increase the blood pressure (Fig 1).

Renal denervation is a novel catheter-based treatment which delivers radiofrequency energy to the lumen of the renal artery, targeting the renal efferent and afferent nerves. This was first recognised as a potential target for treatment of severe hypertension in the 1950s, when surgical denervation was accomplished via removal of a number of thoracic and lumbar sympathetic ganglia, including those supplying the kidneys. A resultant median reduction in blood pressure was observed of 20 $\mathrm{mmHg}$ and a significant improvement in mortality. ${ }^{4}$ However, complications of orthostatic hypotension, urinary and faecal incontinence led to discontinuation of the technique. ${ }^{5}$

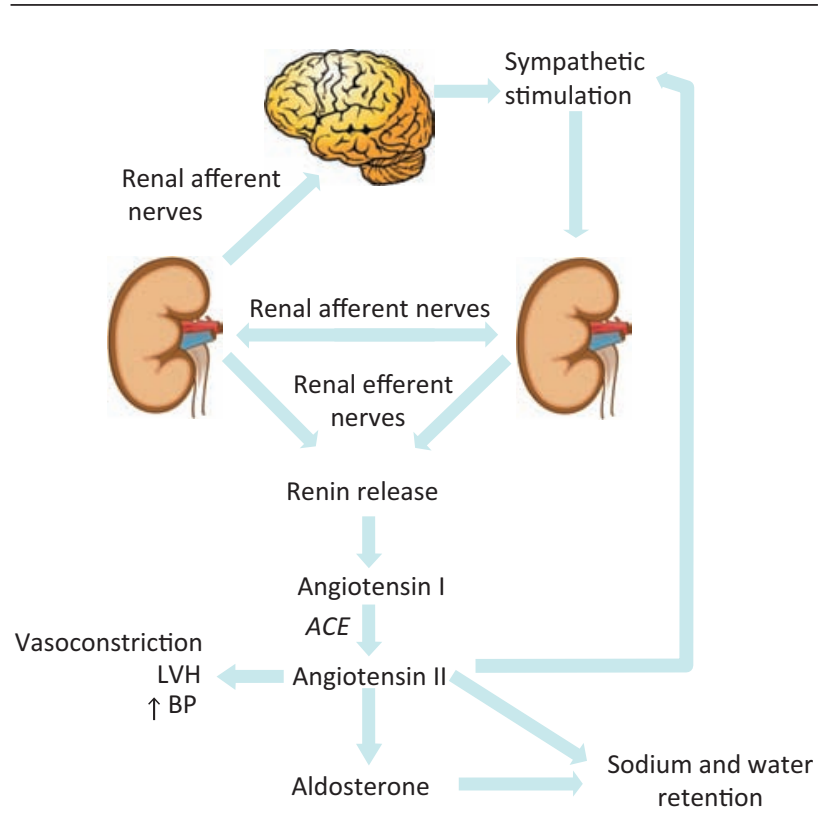

Fig 1. Renal efferent and afferent signalling pathways. 
Development of catheters to allow more selective targeting of the renal sympathetic nerves led to re-examination of the technique of sympathetic denervation. The anatomy of the renal arteries and nerves are well suited for endovascular sympathetic denervation since both afferent and efferent nerves travel together, lying primarily within the adventitia of the renal artery, allowing easy access for radiofrequency ablation. The renal artery is usually accessed via the femoral artery, with a custom designed steerable catheter placed in the ostium of the renal artery and the denervation catheter introduced into the proximal vessel. A variety of catheter designs are available which allow delivery of radio frequency energy to multiple treatment sites in both main renal arteries. Treatment is typically delivered 4-6 times at different locations within the main renal artery, with monitoring of temperature and impedance at each point.

\section{The Symplicity HTN trials}

Symplicity HTN-1, the proof of concept trial, demonstrated large blood pressure reductions in a population of patients with resistant hypertension. ${ }^{1}$ This trial demonstrated that the procedure produced reduction in markers of sympathetic over activity, with a $47 \%$ decrease in norepinephrine spill over one month after the procedure and a 50\% decrease in plasma renin, suggesting an effective reduction in both renal efferent and afferent sympathetic activity.

Symplicity HTN-2 was a multicentre randomised controlled trial of renal denervation in a population of 106 patients with resistant hypertension. ${ }^{6}$ Patients had a systolic blood pressure of $160 \mathrm{mmHg}$ or more (or greater than $150 \mathrm{mmHg}$ in diabetics) and were already compliant with three or more antihypertensive tablets. Patients were randomised to either treatment with renal denervation or usual care. The primary endpoint was office based blood pressure at 6 months. This demonstrated marked improvement with a difference of $32 / 12 \mathrm{mmHg}$ difference in the renal denervation group (178/96 at baseline, falling to $146 / 84$, $\mathrm{p}<0.0001)$ compared with no change in the control group (1/0 mmHg change over a 6 month follow up). Ambulatory blood pressure measurements also demonstrated a significant but less marked improvement, with a mean decrease of $11 / 7 \mathrm{mmHg}$ in the denervation group and no significant change in the control group $(-3 /-1 \mathrm{mmHg})$. This marked reduction in blood pressure appeared sustained at 2 years with no evidence of attenuation of benefit in the original Symplicity HTN-I cohort.

Other improvements suggesting favourable remodelling of the cardiovascular system following renal denervation were also observed, with improved heart rate recovery and blood pressure response to exercise, ${ }^{8}$ a significant decrease in the left ventricular mass and wall thickness in patients with resistant hypertension treated with RDN, and improvements in ejection fraction and diastolic parameters (decrease in lateral E/E' and a shortening of the isovolumic relaxation time) demonstrated by echocardiography. ${ }^{9}$

Symplicity $\mathrm{HTN}-3^{2}$ was the largest trial of renal denervation in hypertension to date, with the important trial design of a sham arm for the control population, in contrast to the previous two Symplicity trials where the control group received usual medical care. This allowed blinding of the study population. The trial enrolled 525 patients over 88 sites; the target population was again patients with severe resistant hypertension, on maximum tolerated doses of at least three anti-hypertensive medications, including a diuretic. The primary end point was the change in office systolic blood pressure at 6 months with secondary efficacy end points of change in mean ambulatory blood pressure and procedural safety.

Both the sham and the renal denervation study groups experienced a significant reduction in blood pressure over the 6 month study period, with a mean change in systolic blood pressure of $-14 \pm 24 \mathrm{mmHg}$ in the denervation group and of $-12 \pm 26 \mathrm{mmHg}$ in the sham arm. This meant that the trial failed to meet its primary end point of a difference of $5 \mathrm{mmHg}$ for superiority of denervation over sham for treatment of hypertension. This was a huge surprise to many in the hypertension community, where denervation was already being offered as a clinical treatment for resistant hypertension in some centres based on the data from the first two Symplicity trials.

Opinions regarding the role and efficacy of renal denervation have been divided following the publication of Symplicity HTN-3. Many scientists and clinicians felt the results suggested that the large blood pressure reductions in the first two Symplicity trials were due to a large placebo effect, perhaps resulting from increased adherence to drug regimens and lifestyle and dietary advice in patients following an invasive procedure. The positive effect on a subject simply by virtue of participation in a research trial is known as the 'Hawthorne effect', where apparent improvements in a condition may occur due to an increase in attention given to the patient, leading to improved compliance with medications or healthier lifestyles. ${ }^{10}$

Of central importance is the question of whether patients in the trial received sufficient therapy to produce denervation. Unlike the early Symplicity HTN-I trial, which measured norepinephrine spillover, no direct or indirect measures of sympathetic activity following the procedure were performed in this trial. Some clinicians have suggested that the 'dose' of denervation may have been insufficient and that the use of 88 centres for 525 patients meant the procedures may have been performed on a learning curve for operators who therefore may have produced incomplete denervation. While such speculation is inevitable, it seems unlikely that the controversy surrounding this technique can be resolved without further trial data, preferably performed with clear measures of sympathetic activity to demonstrate efficacy of denervation.

\section{Renal denervation as a potential treatment of other conditions}

While the published renal denervation trials to date have largely focused on hypertension, other diseases are also characterised by sympathetic over-activity and may therefore potentially benefit from renal denervation. Trials are ongoing in heart failure and these are due to report in the next year or two. Chronic heart failure patients are known to have increased sympathetic tone with subsequent maladaptive hyperstimulation of the renin-angiotensinogenaldosterone system. It is these maladaptive signalling mechanisms that underlie the rationale for treatment with angiotensin-convertingenzyme (ACE) inhibitors, beta blockers and aldosterone antagonists. Norepinephrine spillover from the heart is known to predict mortality and need for transplantation in chronic heart failure, ${ }^{11}$ and so it is hoped that renal denervation would reduce sympathetic overactivity and improve both symptoms and prognosis. 
The REACH trial ${ }^{12}$ in systolic heart failure (due to report in 2015) employs a sham procedure for the control arm, while the Symplicity HF trial ${ }^{13}$ (due to report in 2014/2015) will report measurements of renal norepinephrine spillover post procedure. Both trials will assess procedural safety in these populations as well as changes in symptomatology and objective measures of exercise capacity.

Other small trials have demonstrated potential benefit in arrhythmias, glycaemic control and sleep apnoea. A small double blinded randomised controlled trial of 27 patients with symptomatic paroxysmal or persistent $\mathrm{AF}$ and co-existent drug resistant hypertension demonstrated a greater proportion of patients free of AF 1 year post procedure when treated with pulmonary vein isolation (PVI) and renal denervation compared to PVI alone. ${ }^{14}$ This will be further investigated in the H-FIB trial, a multicentre randomised controlled trial that will assess the effect of adjunctive renal denervation in patients with $\mathrm{AF}$ and significant hypertension planned for first AF-ablation. ${ }^{15}$

Renal denervation has also reported to have a potential role in treatment of insulin resistance. Sympathetic over-activity is known to induce insulin resistance ${ }^{16}$ and two small trials have shown improvement in $\mathrm{HbAlc},{ }^{17}$ fasting glucose, insulin levels and $\mathrm{C}$ peptide levels ${ }^{18}$ post denervation. Patients with sleep apnoea and hypertension treated with renal denervation also demonstrated a significant improvement in their apneahypopnea index (from 16.3 to 4.5 events per hour) and Epworth sleepiness score. ${ }^{17}$ Of note, these patients were unblinded to treatment allocation, so changes may have been due to the Hawthorne effect and improved compliance with treatment.

Patients with polycystic kidney disease commonly experience pain due to renal cysts causing distension of the renal capsule and traction on the renal pedicle. Pain control can be difficult and surgical decompression of large cysts may be required. Laparoscopic renal denervation is an effective treatment for pain refractory to opiod analgesia but requires general anaesthesia. ${ }^{19}$ Catheter based renal denervation may provide an alternative, less invasive treatment. ${ }^{20}$

\section{Summary}

Renal denervation is a novel technique which reduces both efferent and afferent renal sympathetic nerve activity. Initial data showed a dramatic blood pressure reduction in patients with resistant hypertension but the only trial to date conducted against a sham procedure did not show a significant reduction in blood pressure compared to controls. The pathophysiology of many conditions, including heart failure, arrhythmia, sleep apnoea and hyperglycaemia, suggest there may be a role for denervation. However research remains in its early stages in these conditions and further trials are required. For hypertension, while a single randomised controlled trial demonstrated no benefit of renal denervation compared with anti-hypertensive medication, further trials, preferably with assessment of sympathetic activity post procedure, are required to clarify the potential role, if any, of this procedure in clinical practice.

\section{References}

1 Krum H, Schlaich M, Whitbourn R et al. Catheter-based renal sympathetic denervation for resistant hypertension: a multicentre safety and proof-of-principle cohort study. Lancet 2009;373:1275-81.
2 Bhatt DL, Kandzari DE, O'Neill WW et al. SYMPLICITY HTN-3 Investigators. A controlled trial of renal denervation for resistant hypertension. N Engl J Med 2014;370:1393-401.

3 National Institute for Health and Care Excellence. Hypertension: Clinical management of primary hypertension in adults. CG 127 London: National Institute for Health and Care Excellence, 2011.

4 Newcombe CP, Shucksmith HS, Suffern WS. Sympathectomy for Hypertension. Br Med J 1959;1:142-4.

5 Allen TR. Current status of lumbar sympathetctomy. Am Surg 1976;42:89-91.

6 Symplicity HTN-2 Investigators, Esler MD, Krum H et al. Renal sympathetic denervation in patients with treatment-resistant hypertension (The Symplicity HTN-2 Trial): a randomised controlled trial. Lancet 2010;376:1903-9.

7 Symplicity HTN-1 Investigators. Catheter-based renal sympathetic denervation for resistant hypertension: durability of blood pressure reduction out to 24 months. Hypertension 2011;57:911-7.

8 Ukena C, Mahfoud F, Kindermann I et al. Cardiorespiratory response to exercise after renal sympathetic denervation in patients with resistant hypertension. J Am Coll Cardiol 2011;58:1176-82.

9 Brandt MC, Mahfoud F, Reda S et al. Renal sympathetic denervation reduces left ventricular hypertrophy and improves cardiac function in patients with resistant hypertension. J Am Coll Cardiol 2012;59:901-9.

10 McCarney R1, Warner J, Iliffe S et al. The Hawthorne Effect: a randomised, controlled trial. BMC Med Res Methodol 2007;7:30.

11 Petersson M, Friberg P, Eisenhofer G, Lambert G, Rundqvist B. Long-term outcome in relation to renal sympathetic activity inpatients with chronic heart failure. Eur Heart J 2005;26:906-13.

12 Renal Artery Denervation in Chronic Heart Failure Study (REACH), 2012. Available online at http://clinicaltrials.gov/show/ NCT01639378 [Accessed 29 October 2014].

13 Renal Denervation in Patients With Chronic Heart Failure \& Renal Impairment Clinical Trial (SymplicityHF), 2014 (ongoing). Available online at www.clinicaltrials.gov/ct2/show/NCT01392196 [Accessed 29 October 2014].

14 Pokushalov E, Romanov A, Corbucci G et al. A randomized comparison of pulmonary vein isolation with versus without concomitant renal artery denervation in patients with refractory symptomatic atrial fibrillation and resistant hypertension. J Am CollCardiol 2012;60:1163-70.

15 Ahmed H, Miller MA, Dukkipati SR et al. Adjunctive renal sympathetic denervation to modify hypertension as upstream therapy in the treatment of atrial fibrillation (h-fib) study: clinical background and study design. J Cardiovasc Electrophysiol 2013;24:503-9.

16 Mancia G, Bousquet P, Elghozi JL et al. The sympathetic nervous system and the metabolic syndrome. J Hypertens 2007;25:909-20.

17 Witkowski A, Prejbisz A, Florczak E et al. Effects of renal sympathetic denervation on blood pressure, sleep apnea course, and glycemic control in patients with resistant hypertension and sleep apnea. Hypertension 2011;58:559-65.

18 Mahfoud F, Schlaich M, Kindermann I et al. Effect of renal sympathetic denervation on glucose metabolism in patients with resistant hypertension: a pilot study. Circulation 2011;123:1940-6.

19 Resnick M, Chang AY, Casale P. Laparoscopic renal denervation and nephropexy for autosomal dominant polycystic kidney disease related pain in adolescents. J Urol 2006;175:2274-6.

20 Shetty SV, Roberts TJ, Schlaich MP. Percutaneous transluminal renal denervation: a potential treatment option for polycystic kidney disease-related pain? Int J Cardiol 2013;162:e58-9.

Address for correspondence: Dr C Raphael, Department of Cardiology, Royal Brompton Hospital, Sydney Street, SW3 6NP, UK.

Email:c.raphael@rbht.nhs.uk 